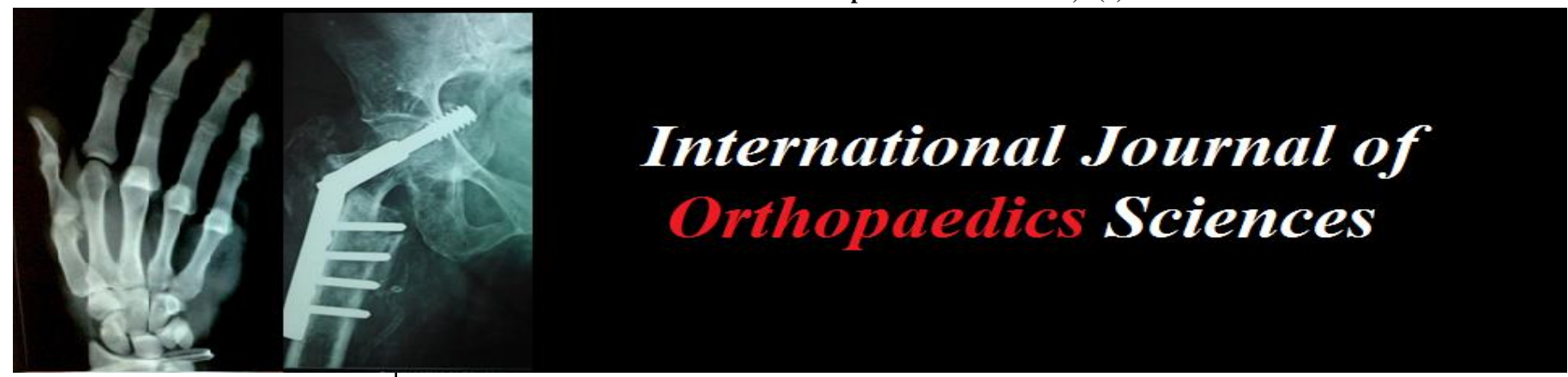

ISSN: $2395-1958$

IJOS 2019; 5(3): 65-68

(C) 2019 IJOS

www.orthopaper.com

Received: 10-05-2019

Accepted: 13-06-2019

Dr. Lakshmeesha T

Associate Professor, Hassan

Institute of Medical Sciences,

Hassan, Karnataka, India

Dr. Ajith Kumar KS

Assistant Professor, Hassan

Institute of Medical Sciences,

Hassan, Karnataka, India

Dr. Vishanth

Senior Resident, Hassan

Institute of Medical Sciences,

Hassan, Karnataka, India

Dr. Kiran SC

Senior Resident, Hassan

Institute of Medical Sciences,

Hassan, Karnataka, India
Correspondence

Dr. Ajith Kumar KS

Assistant Professor, Hassan

Institute of Medical Sciences,

Hassan, Karnataka, India

\section{Study of correlation between clinical findings, radiological and intra operative findings in lumbar disc prolapse}

\author{
Dr. Lakshmeesha T, Dr. Ajith Kumar KS, Dr. Vishanth and Dr. Kiran SC
}

DOI: https://doi.org/10.22271/ortho.2019.v5.i3b.1509

Abstract

Background: Lumbar disc prolapse is one of the common causes of low back pain seen in the working population ${ }^{[1,2]}$. Clinical examination is mainstay of diagnosis, to be confirmed by plain X-ray, invasive radiography like myelography, discography, etc. There is a good correlation between the findings of clinical features and MRI. However, all MRI abnormalities do not correlate with the findings of clinical features.

Study was conducted to correlate between clinical findings, radiological and intra operative findings in lumbar disc prolapsed.

Materials and Methods: Fifty eight cases of symptomatic prolapsed intervertebral disc confirmed by MRI who were not relieved by conservative line of management for a period of 6 weeks were selected for surgical intervention, were studied from 2014 to 2019.

Patients presenting with clinical features of lower limb radiculopathy to the out patient department of orthopedics were screened for inclusion in the study. The patients with clinical diagnosis of lumbar disc prolapse were included in the study. Patients who presented with acute onset of symptoms and were having radicular symptoms for the first time were treated with simple bed rest and analgesics for 6 weeks and patients who had completely recovered were excluded from the study.

Results: There was positive correlation between clinical, MRI findings and intra operative findings in 55 out of 58 and negative correlation in 3 out of 58 patients.

Conclusion: There was positive correlation between clinical, MRI findings and intra operative findings. MRI is a helpful preoperative diagnostic investigation which shows structural changes in the disc the size and the site of the extrusion or protrusion.

Keywords: Lumbar disc prolapse, clinical findings, radiological, MRI, intra operative

\section{Introduction}

Prolapsed intervertebral disc is an important and common cause of low backache.

Clinical examination is mainstay of diagnosis, to be confirmed by plain X-ray, invasive radiography like myelography, discography etc. In last decade or so, non-invasive radiography as CT scan, MRI has taken over the onus of diagnosis of prolapsed intervertebral disc ${ }^{[3]}$.

MRI is considered highly sensitive and specific test for diagnosing prolapsed intervertebral disc, however the problem is over diagnosing the condition ${ }^{[3]}$. Even asymptomatic patients may show disc bulge. Studies have conclusively proved that MR findings of disc degeneration and prolapse can be found normally, especially in aged. MRI has now become universally accepted investigation for prolapsed intervertebral disc ${ }^{[3]}$. We, however, regularly come across situations, when MRI shows diffuse disc bulges, even at multiple levels, which cannot be correlated clinically and when such cases are operated, no significant disc prolapse is found resulting in negative exploration. Various pathoanatomical changes in lumbar disc prolapse can be visualized in MRI. However, the clinical significance of MRI findings is still controversial.

Despite the fact that MRI is done routinely for patients with suspected intervertebral disc prolapse, one is not sure which of the MRI findings are clinically relevant, and have diagnostic as well as prognostic value. Milette et al. ${ }^{[4]}$ found that loss of disc height or abnormal signal intensity is highly predictive of symptomatic tears and the presence of a disc bulge or 
protrusion does not have additional significance. Beattie et al. ${ }^{[5]}$ found that the presence of disc extrusion and severe nerve root compression at one or multiple sites is strongly associated with distal leg pain. However, Rankine et al. ${ }^{[6]}$ in their study opined that pain drawing is not a good predictor of nerve compression on magnetic resonance imaging with poor correlation. Borenstein ${ }^{[7]}$ and others clearly opined that MRI findings were not predictive of the development or duration of low back pain and that clinical correlation is essential.

There is a good correlation between the findings of clinical features and MRI. However, all MRI abnormalities do not correlate with the findings of clinical features. The presence of centrolateral protrusion of the disc and the extrusion with gross neural foramen compromise are associated with the clinical features. Bulging of the disc with compression of thecal sac or central protrusions and extrusions without significant neural foramen compromise are clinically insignificant.

So the dilemma lies, whether to operate these patients with MRI findings and if so, how extensive the surgery should be? It is here, that importance of clinical correlation with MRI findings has to be made so that "negative explorations" can be avoided.

So we decided to study the correlation between clinical features, MRI findings and intra operative findings in lumbar disc prolapse and to know about its significance.

The aim of our study is to emphasize the fact that MRI findings should always be correlated with clinical findings to decide about surgical intervention.

\section{Material and Methods}

The patients with low back ache who attended the orthopaedic opd in hims, Hassan were evaluated and treated initially with conservative methods. Out of which fifty eight cases of symptomatic prolapsed intervertebral disc confirmed by MRI who were not relieved by conservative line of management for a period of 6 weeks were selected for surgical intervention, were studied from 2014 to 2019.

Patients presenting with clinical features of lower limb radiculopathy to the outpatient department of orthopedics were screened for inclusion in the study. The patients with clinical diagnosis of lumbar disc prolapse were included in the study. Patients who presented with acute onset of symptoms and were having radicular symptoms for the first time were treated with simple bed rest and analgesics for 6 weeks and patients who had completely recovered were excluded from the study.

The indications for surgery were:

1. Pain for at least six weeks in an adult male/female aged between $20-70$ years

2. Persistent pain unrelieved by conservative treatment. Painful radiculopathy and neurological deficit.

3. Deteriorating neurology.

4. Cauda equina syndrome.

\section{Exclusion criteria}

- Patients with low back pain lasting for less than 6 weeks,

- Osseous cause

- Bony canal stenosis

- Individuals who had undergone previous discectomy and epidural steroid injection procedures

- Infections of spine and tumors of spine were excluded from study.

- $\quad$ Age less than 20 years and more than 70 years.

On admission, all patients were evaluated by taking detailed history, thorough general and neurological examination with particular attention to lower limbs. Each patient was evaluated for the distribution of pain and the presence of neurological features. The distribution of pain at the dermatomal level was pointed out.

The diagnosis was confirmed with radiographic imaging. Lateral and antero-posterior plain radiographs of lumbar spine were taken to evaluate osseous anatomy and alignment. Later magnetic resonance imaging was done to confirm the disc herniation, to grade the disc herniation and also to rule out other possible causes of back pain such as infection in spine, tumours of spine and intra-abdominal visceral causes which will have major bearing with respect to treatment and inclusion into the study.

MRI was designated as significant when compression of thecal sac and adjoining nerve root was present. Evaluation of MRI of the lumbosacral spine including degeneration of the disc, the extent of disc prolapse, nerve root contact/ compression, neural foramen compromise, and other findings (hypertrophy of ligamentum flavum or facet joint, spondylolisthesis, stenosis of the canal) were done.

Preoperatively, the type of surgical procedure was decided on the basis of clinical correlation with MRI findings. Haematological (viz., Hb, TC, DC, ESR, Platelet counts), biochemical (blood sugar, blood urea, serum creatinine), serological (HIV, HBsAg) investigation were done.

All patients underwent conventional lumbar discectomy. Intra-operatively disc was excised when bulge was significant and gave doughy feeling on palpation.

Inflammation and displacement of adjoining nerve root is important indication of pathological bulge. Intra-operative findings were noted in terms of disc bulge, its level, status of nerve root, and these were compared with MRI findings

Patients were followed up at regular intervals for improvement in pain and neurological status, any complications, and when they were able to go back to original schedule of work. Results were graded as surgical success and failure.

\section{Observations and Results}

Fifty eight patients operated from 2015 to 2019 were included. The age ranged between 20 to 70 years. The majority were males. Follow up ranged from six to 24 months.

Of the 58 patients, $45(77.5 \%)$ patients were in between $30-$ 50 years. $32(55 \%)$ patients were male and 26(45\%) were female.

In our study $37(63 \%)$ patients were labourers and 14 $(24.13 \%)$ patients were housewives.

On clinical examination SLRT was positive in all the patients out of which in $34(58.6 \%)$ patients it was bilaterally positive and in $24(41.3 \%)$ of them it was unilaterally positive with left sided SLRT positive in 14 patients and right sided positive in 10 patients.

Neurological involvement was present in $42(72.4 \%)$ patients and it was absent in remaining $16(27.6 \%)$ patients.

MRI findings of disc bulge, single or multiple, compression of nerve root and thecal sac and canal stenosis were noted. Over $72.4 \%$ patients with MRI findings of protrusion or extrusion of disc with nerve root compression had neurogical involvement. 44 patients had single level disc prolapse on MRI out of which most common level was L4-L5 seen in 35 patients, L5-S1 seen in 7 patients and L3-L4 seen in 2 patients. 14 patients had 2 level disc prolapse most common level among them was L4-L5 \& L5-S1. 
There was positive correlation between clinical, MRI findings and intra operative findings in 55 out of 58 and negative correlation in 3 out of 58 patients.ie all the patients were clinically examined, SLRT and neurological involvement were noted and clinical level of involvement were noted and then patients were subjected to MRI and findings were noted and then all patients underwent lumbar discectomy and intra operative findings in terms of level, site, stage and nerve root or cord compression were noted. When clinical, radiological and intra operative findings were correlated the level of involvement were same in 55 of 58 patients and level differed in 3 of 58 patients.

MRI was accurate in determining disc prolapse level except for three patients in present series, We evaluated the correlation between clinical, MRI \& intra operative findings. In most cases, pain distribution could be correlated with a particular level making it easy to compare the clinical and MRI levels. Our results show that there is a very good correlation between the clinical level, MRI level and intra op level.

Complications encountered were dura tear in three, root damage in one, low backache without radiculopathy in 21, Complications were managed appropriately.

\section{Discussion}

Lumbar disc protrusions are more frequent in middle-aged people from third to the fifth decade of life. Lumbar disc protrusions are more frequent in middle-aged people from third to the fifth decade of life. The herniation of the lumbar disc is more common at L4/5 and L5/S1. Less than $5 \%$ of it occurs at L1/2, L2/3 and L3/4. Fracture of intravertebral disc may occur following injury ${ }^{[8]}$. earliest symptom of the root involvement in a lumbar disc prolapse is radiating pain along the sciatic nerve (sciatica) ${ }^{[9]}$. In our study the age ranged between 20 to 70 years. Of the 58 patients, $45(77.5 \%)$ patients were in between $30-50$ years compared to other study ${ }^{[10]}$ in which L4-L5 (58.7\% ) is the most level and the next common level was L5-S1(36\%) this is similar with other studies, disc herniation an end-plate defects show little correlation with age and the most commonly affect only the lower lumbar levels mainly L4-5 intervertebral disc space was the most commonly afflicted level, and it is similar to another study ${ }^{[11]} 56 \%$ (28patients) had herniation at L4-5 level, 44\% (22 patients) of them had herniation at L5-S1 level, which is near to the study done in 2007 (The mean age in our study was 43 years range ${ }^{[12]}$ from 21 to 65 years), and it is higher than other studies [13] Baldwin NG mean age of 34.96 years (range 21-72 years) ${ }^{[14,}$ ${ }^{15]}$. Battie MC et al. Weber H.

In our study the majority were males. $32(55 \%)$ patients were male and $26(45 \%)$ were female, which is comparable to study which includes 31 males $(62 \%)$ and 19 females, other study ${ }^{[15]}$ reported that male patients was $75 \%$ and the female patients was $25 \%$.

In our study $37(63 \%)$ patients were labourers and 14 $(24.13 \%)$ patients were housewives, which were comparable to other studies.

According to most literature, the common presentation of lumbar disc herniation was low back pain and sciatica. According to most literature, Low back pain was reported in $99 \%$ cases and leg pain/sciatica in $79 \%$ cases ${ }^{[16]}$. In our study, all the cases presented with low back pain and sciatica. On clinical examination SLRT was positive in all the patients out of which in $34(58.6 \%)$ patients it was bilaterally positive and in $24(41.3 \%)$ of them it was unilaterally positive with left sided SLRT positive in 14 patients and right sided positive in
10 patients.

Findings were comparable to a study ${ }^{[16]}$, in which all the cases presented with low back pain and sciatica. Right sciatica was found in $46.3 \%$ of cases whereas left sciatica was in $37.0 \%$ cases. Bilaterally sciatica was present in $16.7 \%$ patients which are nearly similar to another study ${ }^{17}$. Right sciatica was in $51.0 \%$, left sciatica in $43 \%$ and bilateral sciatica in $6 \%$ of the PLID cases.

In our study the Neurological involvement was present in 42 (72.4\%) patients and it was absent in remaining 16 (27.6\%) patients. Compared to other study ${ }^{[18]}$ in which the presence of weakness in one or both extensor hallucis longus was reported by $42.9 \%$ participants, $35 \%$ reported the sensation of pin and needles and $37.4 \%$ reported numbness and sphincter dysfunction was present in $6.2 \%$ cases.

Magnetic Resonance Imaging is a universally accepted investigation for space occupying lesion in spinal canal. It provides exact localization of the lesion as intradural, intramedullary, or extradural. In one series ${ }^{[10]}$ of seventy patients MRI found disc prolapse at L45 levels. This clearly shows the sensitivity of MRI to diagnose even sub-clinical disc prolapse. MRI has 92 percent sensitivity, specificity and accuracy in diagnosing protruded disc. This accuracy is less for subligamentous and transligamentous extruded disc. High resolution MRI can detect disc disease and is specific in characterizing various sub groups of disc herniation, especially sequestrated.

The clinical features and the MRI findings of the level of disc prolapse correlate well which might not be considered as essential for the clinical diagnosis. But MRI is mandatory when someone is planned for surgery. Not all the lesions identified in MRI have symptoms. In a study ${ }^{[10]}$ out of 113 levels of disc lesions, only 65 were symptomatic. However, MRI is a sensitive test for identifying disc lesions but it is not specific. MRI finding with neural foramen compromise and nerve root compression are likely to be more symptomatic than those without neural foramen compromise.

Another important finding is that single level had a higher incidence i.e. 89 percent of surgically significant disc prolapse, than two level (85 percent) and multiple level discs prolapse (56 percent). Moreover lateral disc herniation was more significant than central or paramedian type as incidence of surgically significant disc was 100 percent in lateral disc herniation compared to 83 percent in paramedian and 62 percent in central type. Central disc herniation or rather disc bulge especially at multiple levels is a common finding in MRI. This probably causes an inflammatory cycle to set in leading to symptoms, more so in compromised spinal canal. In such patients a simple decompression procedure without excision of the disc is sufficient.

The indications for surgery in prolapsed intervertebral disc are standard and we corroborated our clinical findings with MRI findings.

In our series MRI was accurate in determining disc prolapse level except for three patients in present series, We evaluated the correlation between clinical, MRI \& intra operative findings. In most cases, pain distribution could be correlated with a particular level making it easy to compare the clinical and MRI levels. Our results show that there is a very good correlation between the clinical level MRI level and intra op level.

There was positive correlation between clinical, MRI findings and intra operative findings in $55(94,8 \%)$ out of 58 and negative correlation in $3(5.17 \%)$ out of 58 patients. ie all the patients were clinically examined, patients were subjected to 
MRI and all patients underwent lumbar discectomy and intra op findings were noted. When clinical, radiological and intra operative findings were correlated the level of involvement were same in 55 of 58 patients and level differed in 3 of 58 patients.

MRI is a helpful preoperative diagnostic investigation which shows structural changes in the disc the size and the site of the extrusion or protrusion. However, these image are not useful in predicting neurological deficit and therefore should not be used as an indication for surgery unless there is a strong correlation with the clinical findings. The findings were comparable to other study ${ }^{[10]}$ in which $34(63.0 \%)$ patients had signs and symptoms of PLID at the level L4/5, 53.7\% at L5/S1 and 11.1 cases at L3/4 level; which on MRI was found to be $68.5 \%, 46.3 \%$ and $11.1 \%$ respectively, showing good agreement for $\mathrm{L} 4 / 5$ and L5/S1 (k-value 0.795 an 0.705 respectively) and very good agreement for L3/4 level ( $\mathrm{p}$ value 0.812 ), between the two modalities of diagnosis in detecting level of disc prolapse. MRI detected herniation at an L2/3 level in 1 case which could not diagnose clinically. So for evaluation of high lumbar disc prolapse, MRI is very essential.

Complications encountered were dura tear in three, root damage in one, low backache without radiculopathy in 21 . Complications were managed appropriately. In most it was mild and did not interfere with daily activities. This is comparable to $30 \%$ complication rate in a study reported by Spangfort et al. ${ }^{[19]}$ It is important to explain the patients preoperatively that disc surgery may lead to low backache regardless of the technique used. Recurrent herniation is a possibility where again MRI is important for its accuracy to distinguish scar tissue and disc material.

\section{Conclusion}

There was positive correlation between clinical, MRI findings and intra operative findings. MRI is a helpful preoperative diagnostic investigation which shows structural changes in the disc, the size and the site of the extrusion or protrusion. However, these image are not useful in predicting neurological deficit and therefore should not be used as an indication for surgery unless there is a strong correlation with the clinical findings. The indications for surgery in prolapsed intervertebral disc are standard and we corroborated our clinical findings with MRI findings.

\section{References}

1. Postacchini F. Management of herniation of lumbar disc. J Bone Joint Surg Br. 1999; 81:567-76.

2. Vroomen PC, de Krom MC, Wilmink JT. Pathoanatomy of clinical findings in patients with sciatica: A magnetic resonance imaging study. J Neurosurg Spine. 2000; 92:135-41.

3. Wiesel SW. Lumbar spine imaging; role in clinical decision making. J Am Acad Orthop Surg. 1996; 4:238248.

4. Milette PC, Fontaine S, Lepanto L, Cardinal E, Breton G. Differentiating lumbar disc protrusions, disc bulges, and discs with normal contour but abnormal signal intensity: Magnetic resonance imaging with discographic correlations. Spine (Phila Pa 1976). 1999; 24:44-53.

5. Beattie PF, Meyers SP, Stratford P, Millard RW, Hollenberg GM. Associations between patient report of symptoms and anatomic impairment visible on lumbar magnetic resonance imaging. Spine (Phila $\mathrm{Pa}$ 1976). 2000; 25:819-28.
6. Rankine JJ, Fortune DG, Hutchinson CE, Hughes DG, Main CJ. Pain drawings in the assessment of nerve root compression: A comparative study with lumbar spine magnetic resonance imaging. Spine (Phila $\mathrm{Pa}$ 1976). 1998; 23:1668-76.

7. Borenstein DG, O'Mara JW Jr, Boden SD, Lauerman WC, Jacobson A, Platenberg $\mathrm{C}$ et al. The value of magnetic resonance imaging of the lumbar spine to predict low-back pain in asymptomatic subjects: A sevenyear follow-up study. J Bone Joint Surg Am. 2001; 83:1306-11.

8. Ahsan M, Sakeb N, Zaman N, Nur-E-Jannat S. Management of traumatic spinal column injury: A tertiary hospital experience. Bangabandhu Sheikh Mujib Med Univ J. 2015; 8:95-104.

9. Goel A, Pandaya S. Lumbar disc protrusion. In: Textbook of neurosurgery. Ramamurti B, Tandon PN (eds). 2nd ed. New Delhi, Churchill Livingstone, 1996.

10. Association between clinically diagnosed lumbar intervertebral disc prolapse and magnetic resonance image findings. Md. Habibur Rahman, K. M. Tarikul Islam, Md. Rokibul Islam, Moududul Haque, Haradhon Devnath, Mohammad Afzal Hossain and Kanak Kanti Barua. Al-Kindy College Medical Journal. 2015; 11(1):25-27.

11. Prospective study Correlation between clinical and MRI findings in disc herniation at lumbo-sacral region athesis submitted to the iraqi commission for medical specialization as a partial fulfillment of the requirments for the degree of fellowship of fractures and orthopaedic surgery, dr. mustafa saad gahnim, 2007.

12. Baldwin NG. Lumbar disc disease: the natural history. Neurosurg Focus. 2002; 15;13(2):E2. http://www.ncbi.nlm.nih.gov/pmc/articles/

13. Battie MC, Videman T, Parent E. Lumbar disc degeneration: Epidemiology and genetic influences. Spine, 2004.

14. Weber H. The natural history of disc herniation and the influence of intervention. Spine. 1994; 19:2233-2238

15. Weir BK. Prospective study of 100 lumbosacral discectomies. J Neurosurg. 1979; 50:283-89.

16. Pappas CT, Harrington $\mathrm{T}$, Sonntag VK. Outcome analysis in 654 surgically treated lumbar discherniations. Neurosurgery. 1992; 30:862-66.

17. Kortelainen P, Puranen J, Koivisto E, Lahde S. Symptoms and signs of sciatica and their relationto the localization of the lumbar disc herniation. Spine (Phila PA 1976). 1985; 10:88.

18. Beattie PF, Meyers SP, Stratford P, Millard RW, Hollenberg GM. Associations between patient report of symptoms and anatomic impairment visible on lumbar magnetic resonance imaging. Spine (Phila PA 1976). 2000; 25:819-28.

19. Spangfort EV. Prolapse intervertebral disc Acta orthop Scand Suppl. 1972; 142:65. 